\title{
Effect of continuity of care on anticoagulant therapy and quality of life after heart valve replacement: a systematic review and meta- analysis
}

\author{
Xia Yuan ${ }^{1,2 \#}$, Yihong Chen ${ }^{1 \#}$, Yu Zhuang ${ }^{2}$, Wei Qin ${ }^{1}$, Ying Lin ${ }^{1}$ \\ ${ }^{1}$ Department of Nursing, Zhongshan Hospital, Fudan University, Shanghai, China; ${ }^{2}$ School of Nursing,Fudan university, Shanghai, China \\ Contributions: (I) Conception and design: X Yuan; (II) Administrative support: W Qin, Y Lin; (III) Provision of study materials or patients: Y Chen; (IV) \\ Collection and assembly of data: Y Zhuang; (V) Data analysis and interpretation: X Yuan, Y Chen; (VI) Manuscript writing: All authors; (VII) Final \\ approval of manuscript: All authors. \\ \#These authors contributed equally to this work. \\ Correspondence to: Wei Qin; Ying Lin. Zhongshan Hospital, Fudan University, Shanghai, China. \\ Email: qin.wei@zs-hospital.sh.cn; lin.ying@zs-hospital.sh.cn.
}

\begin{abstract}
Backgrounda Valvular heart disease (VHD) is a leading cause of heart diseases and death, and heart valve replacement (HVR) plays a fundamental role in treating valve disease and improving the function of valves. The lack of continuity of care (CC, also known as transitional care) for postoperative patients after discharge often results in a variety of complications, bringing severe pain to the patients and diminishing their quality of life.
\end{abstract}

Methods: We systematically searched for relevant randomized controlled trials (RCTs) in Cochrane databases, PubMed, Embase, CINAHL, Web of Science, China National Knowledge Infrastructure (CNKI), Chinese Biomedical Literature Database (CBM), Chongqing VIP, and Wanfang Data. The methodological quality of the included articles was assessed using the Cochrane Handbook for Systematic Reviews of Interventions. The meta-analysis was conducted with RevMan 5.2 software.

Results: A total of 14 RCTs (involving 1,825 patients), all in Chinese language, were included in the analysis, including 920 patients in the CC group and 905 patients in the control group (receiving the routine care). Meta-analysis showed that, compared with the control group, the CC group had significantly higher anticoagulation therapy adherence $[\mathrm{RR}=1.36,95 \% \mathrm{CI}$ : $(1.25,1.48), \mathrm{P}<0.00001]$, higher awareness of anticoagulation [RR $=1.14,95 \% \mathrm{CI}$ : $(1.09,1.18), \mathrm{P}<0.0001]$, and lower incidences of anticoagulation complications and adverse events $[\mathrm{RR}=0.24,95 \% \mathrm{CI}:(0.17,0.35), \mathrm{P}<0.00001]$. In addition, the 5 domains reflecting quality of life including physical domain [MD =2.38, 95\% CI: $(1.30,3.46), \mathrm{P}<0.0001]$, psychological domain $[\mathrm{MD}=2.92,95 \% \mathrm{CI}$ : $(1.47,4.37), \mathrm{P}<0.0001]$, levels of independence $[\mathrm{MD}=4.03$, 95\% CI: $(1.04,7.01), \mathrm{P}=0.008]$, social relationships [MD=2.56, 95\% CI: $(1.81,3.32), \mathrm{P}<0.00001]$, and environment $[\mathrm{MD}=4.15,95 \% \mathrm{CI}$ : $(1.16,7.14), \mathrm{P}=0.007]$ were also significantly improved.

Discussion: Our results showed CC can effectively improve patients' anticoagulation therapy adherence and raise their awareness about medications, reduce the incidences of complications and adverse events, and thus improve the patients' quality of life. However, only a limited number of high-quality RCTs were included in our current analysis, and studies with more rigorous designs are warranted to further validate the impacts of CC on anticoagulation therapy adherence and quality of life after HVR.

Keywords: Continuity of care; anticoagulant therapy; quality of life; meta-analysis

Submitted Mar 24, 2021. Accepted for publication May 19, 2021.

doi: 10.21037/apm-21-1167

View this article at: http://dx.doi.org/10.21037/apm-21-1167 


\section{Introduction}

Valvular heart disease (VHD) is a series of clinical syndromes caused by anatomical or functional abnormalities of the heart valves and their surrounding tissues due to various acquired pathologies or congenital developmental malformations, which result in single or multiple valve stenosis or incomplete closure. VHD is considered to be a major cause of heart disease and death (1). The incidence of VHD is 2.5 to 3.2 per 1,000 person-years in China (2). Heart valve replacement (HVR) is a surgical procedure to restore the normal function of heart valves by replacing the damaged ones with artificial heart valves (3). Continuity of care (CC) refers to the extended nursing services offered by specific medical personnel to the discharged patient after HVR. If necessary, home follow-up services can be arranged to enable the medical staff to offer consultations to the patients (4-6). It has been found that, due to the lack of knowledge on anticoagulation and nursing instructions, the postoperative patients after discharge often suffer from a variety of complications, such as thromboembolism, angina pectoris, pulmonary infection, and bleeding, bringing severe pain to the patients and diminishing their quality of life (7). In China, conventional nursing education is typically delivered upon discharge. Although the benefits of CC in VHD patients have been demonstrated in literature, its efficacy remains controversial due to the limited inputs from health care professionals. The aim of this study was to investigate of impacts of $\mathrm{CC}$ on anticoagulant therapy and quality of life after HVR and thus inform the promotion of $\mathrm{CC}$ in clinical settings.

We present the following article in accordance with the PRISMA reporting checklist (available at http://dx.doi. org/10.21037/apm-21-1167).

\section{Methods}

\section{Search strategy}

We systematically searched for relevant clinical controlled trials until March 2020 in Cochrane databases, PubMed, Embase, CINAHL, Web of Science, China National Knowledge Infrastructure (CNKI), Chinese Biomedical Literature Database (CBM), Chongqing VIP, and Wanfang Data. The Chinese keywords included heart valve, aortic valve insufficiency, aortic stenosis, mitral valve atresia, mitral valve stenosis, tricuspid stenosis, surgery, cardiovascular surgery, replacement, continuous nursing, continuity of care, extended care, and discharge plan. English keywords comprised a combination of subject terms with a wide range of free-text terms, which included cardiac valve/heart valve disease, surgery/operation/replacement/heart surgical procedure, and continuation of care/continuity of care/ extended care/discharge plan. Boolean operators were used during searching.

\section{Inclusion and exclusion criteria}

\section{Inclusion criteria \\ Study types}

Randomized controlled trials, in English or Chinese, were enrolled, regardless of whether the study mentioned concealed allocation or blinding.

\section{Subjects}

Patients who had been discharged from hospital after HVR were enrolled.

\section{Interventions}

The control group received conventional treatment and nursing, which included instructions at admission, routine preparation, and health education before and after the surgery, and routine instructions upon discharge. The CC group received $\mathrm{CC}$ in addition to the routine treatment and nursing. The main content of CC included the following: (I) health education focusing on disease-related knowledge during hospitalization; (II) a detailed nursing plan after discharge; (III) rehabilitation instructions; and (IV) telephone visits, home visits, or internet-based visits (e.g., via social media). The duration of the intervention was longer than 1 month.

\section{Outcome measures}

The outcome measures were the following: (I) anticoagulation therapy adherence: patients' adherence to anticoagulation therapy was evaluated by using a self-made scale based on consulting relevant literature and experts, which clearly included the continuity of medication, drug administration of the appropriate dose at the right time, regular tests for relevant indicators, reasonable diets, and self-monitoring. The instrument used a 4-point Likert scale (strongly agree $=4$, agree $=3$, disagree $=2$, strongly disagree $=1$ ), with 20 indicating the highest score possible. A higher score indicates better adherence to anticoagulation therapy, a score of 16 or more is considered good adherence, and a score of 16 or less is considered poor adherence. (II) Incidence of anticoagulation-associated complications and adverse events: The complications and adverse events (e.g., hemorrhage, embolism, and thrombosis) of anticoagulation were observed and recorded in both groups. Hemorrhage 
was confirmed by chest X-ray/ultrasound or head computed tomography (CT). Other organic conditions that may cause bleeding were ruled out. Cerebral embolism was confirmed by head CT, and peripheral embolism was confirmed by color Doppler ultrasound. Thrombosis was confirmed by color Doppler echocardiography and other examinations. (III) Awareness about anticoagulation therapy: a questionnaire was designed according to the actual situation, including the importance of anticoagulation therapy, the values of regular tests for prothrombin time (PTT) and international normalized ratio (INR), adverse effects and complications, and lifestyle. The evaluation of awareness was divided into 3 levels: fully aware, partially aware, and not aware. The anticoagulation awareness rate $=$ (fully aware + partially aware)/total number. (IV) Quality of life: the World Health Organization Quality of Life Scale Brief Version (WHOQOL-BREF) was used to assess the survival status of patients in both groups 12 months after discharge. The WHOQOL-HIV BREF is a 26-item tool distributed under 5 main domains comprising physical domain, psychological domain, levels of independence, social relationships, and environment. Individual items are rated on a 5 -point Likert scale where 1 indicates low, negative perceptions; and 5 indicates high, positive perceptions.

\section{Exclusion criteria}

The exclusion criteria included (I) duplicate publications, (II) incomplete baseline data, (III) incomplete reporting, (IV) poor quality of the literature or studies in which the statistical data could not be translated or applied, and (V) self-controlled trials.

\section{Screening and quality evaluation of studies published}

\section{Literature screening and information extraction}

The procedure for literature screening and quality evaluation was as follows: (I) duplicated articles were removed using NoteExpress software (version 3.2; Aegean Software Corp.); (II) two researchers independently read the titles and abstracts and manually removed the articles that did not meet the inclusion criteria of the present study; (III) the articles were further screened through a reading of the full text and excluded or included according to the inclusion and exclusion criteria of the given study, with any disagreement being settled by consultation with the supervisor; (IV) Two researchers independently extracted information including first author(s), publication year, follow-up duration, study method, subject characteristics, interventions, and outcome measures. A formal information extraction form was submitted after an agreement was reached between these two researchers.

\section{Quality evaluation}

The two researchers evaluated the methodological quality of the studies included according to the RCT bias risk assessment tool recommended by the Cochrane systematic review guidelines (8). In the case of disagreement, the two researchers discussed or resolved the issue by consulting a third researcher. The evaluation included the following 7 items: (I) random sequence generation; (II) allocation concealment; (III) performance bias (blinding of participants and personnel); (IV) detection bias(blinding of outcome assessment) (V) attrition bias (incomplete outcome data); (VI) reporting bias (selective reporting); and (VII) other bias. Each item was rated as "low risk of bias," "unclear risk of bias," or "high risk of bias." If all the above criteria were met, the possibility of bias was considered to be low and the quality level was "A"; if only some of the criteria were met, the possibility of bias was considered to be moderate and the quality level was "B"; and if none of the criteria were met, the possibility of bias was considered to be high and the quality level was "C".

\section{Statistical analysis}

Meta-analysis was performed using the RevMan 5.2 software. Count data are presented with relative risks (RRs), ad measure data are presented with weighted mean differences (WMDs), and all the effect measures are expressed as $95 \%$ confidence intervals (CIs). The heterogeneity analysis was performed using the $\chi^{2}$ test. When $\mathrm{I}^{2}<50 \%$ and $\mathrm{P} \geq 0.1$, the studies were considered slightly heterogeneous or no-heterogeneous, and a fixed-effects model was used; when $\mathrm{I}^{2} \geq 50 \%$ and $\mathrm{P}<0.1$, possibility of heterogeneity was considered, and a randomeffects model was used. A P value of $<0.05$ was considered statistically significant.

\section{Results}

\section{Results of literature retrieval}

In total, 1,610 records (753 in Chinese and 857 in English) were retrieved in the initial electronic search. After removal of 120 duplicate articles, 1,400 documents were further 


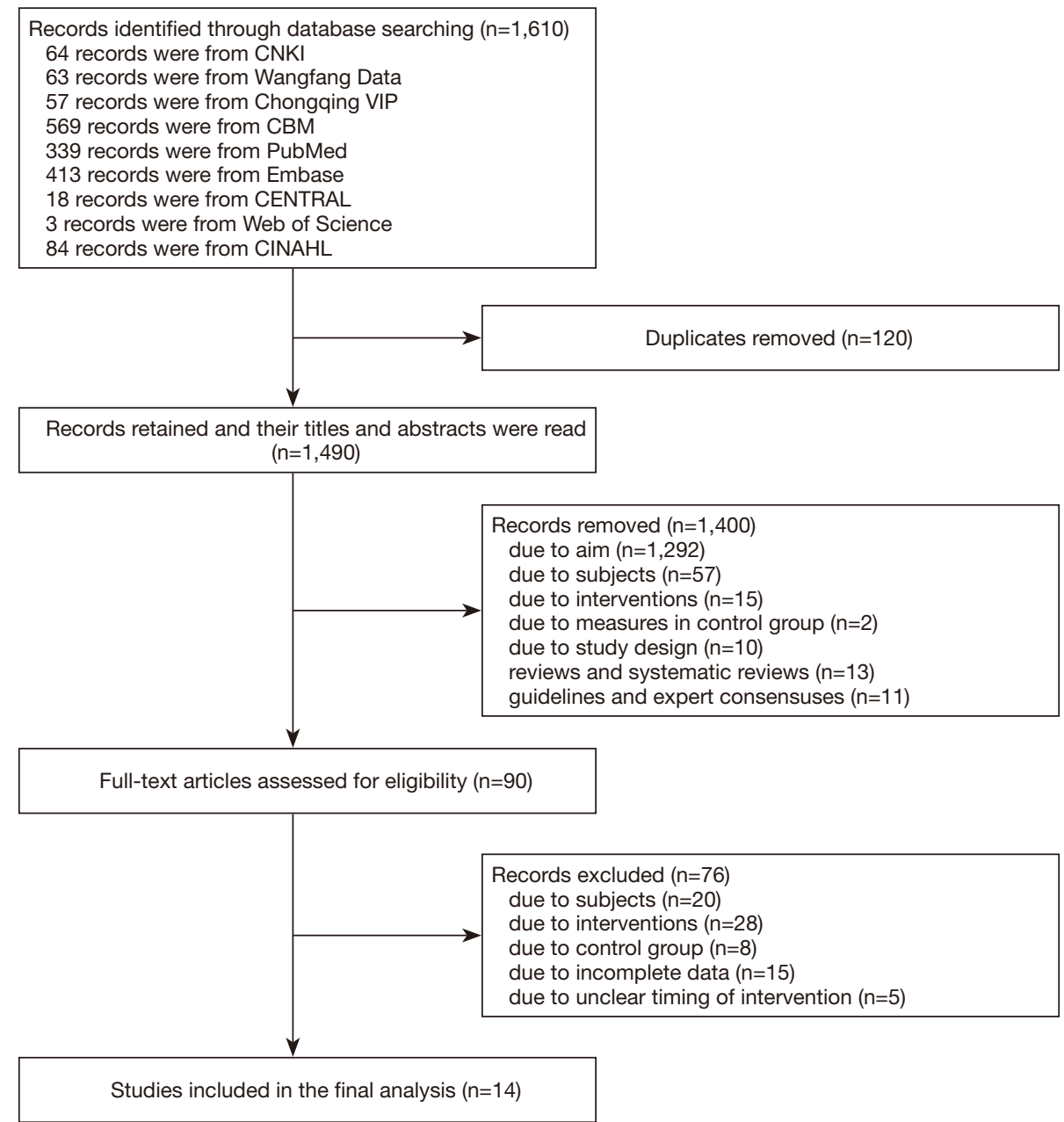

Figure 1 Flow diagram depicting the process of study selection.

removed according to the inclusion and exclusion criteria after reading the titles and abstracts. Subsequently, the full texts were downloaded and read, and 76 articles that did not meet the inclusion criteria were excluded. Finally, 14 randomized controlled trials (RCTs) (9-22) involving 1,825 patients ( 920 and 905 patients in each group) entered the final analysis. All were Chinese-language articles. A flowchart depicting the selection process is provided in Figure 1.

\section{General characteristics of the included articles}

These 14 Chinese-language articles were published between 2014 and 2019. The general characteristics of these articles are summarized in Table 1.

\section{Quality levels of the included literature}

All 14 articles were grade B (Table 2).

\section{Results of the meta-analysis}

\section{Compliance with anticoagulation therapy}

In all, 7 of the included studies used anticoagulation adherence as an outcome measure $(9,11,12,16-18,22)$; among these, 3 reported patients' anticoagulation adherence at 3 months, 6 months, and 1 year postoperatively $(9,11,16)$, 1 reported patients' anticoagulation adherence at 6 months and 1 year postoperatively (12), and 3 reported patients' 


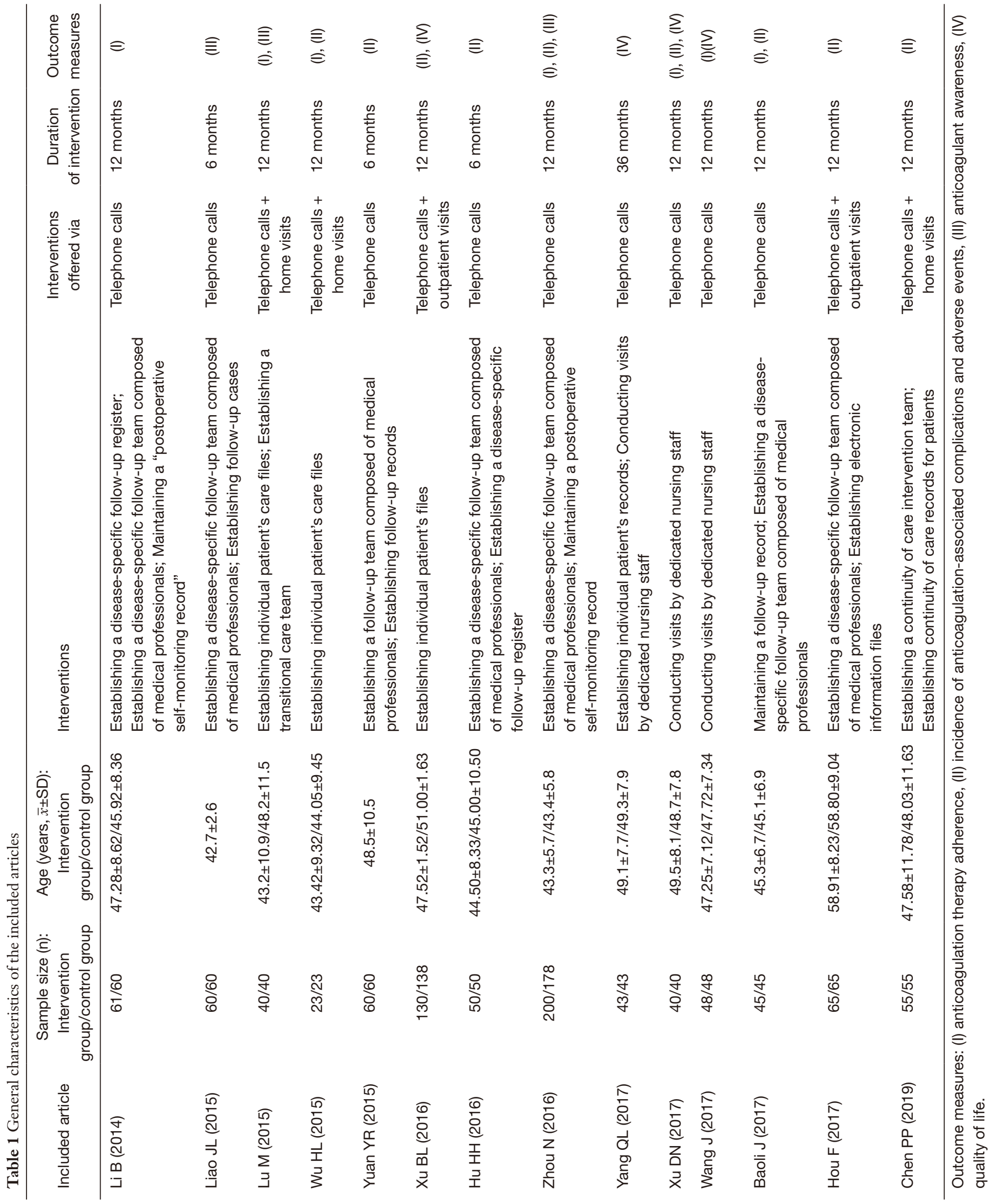




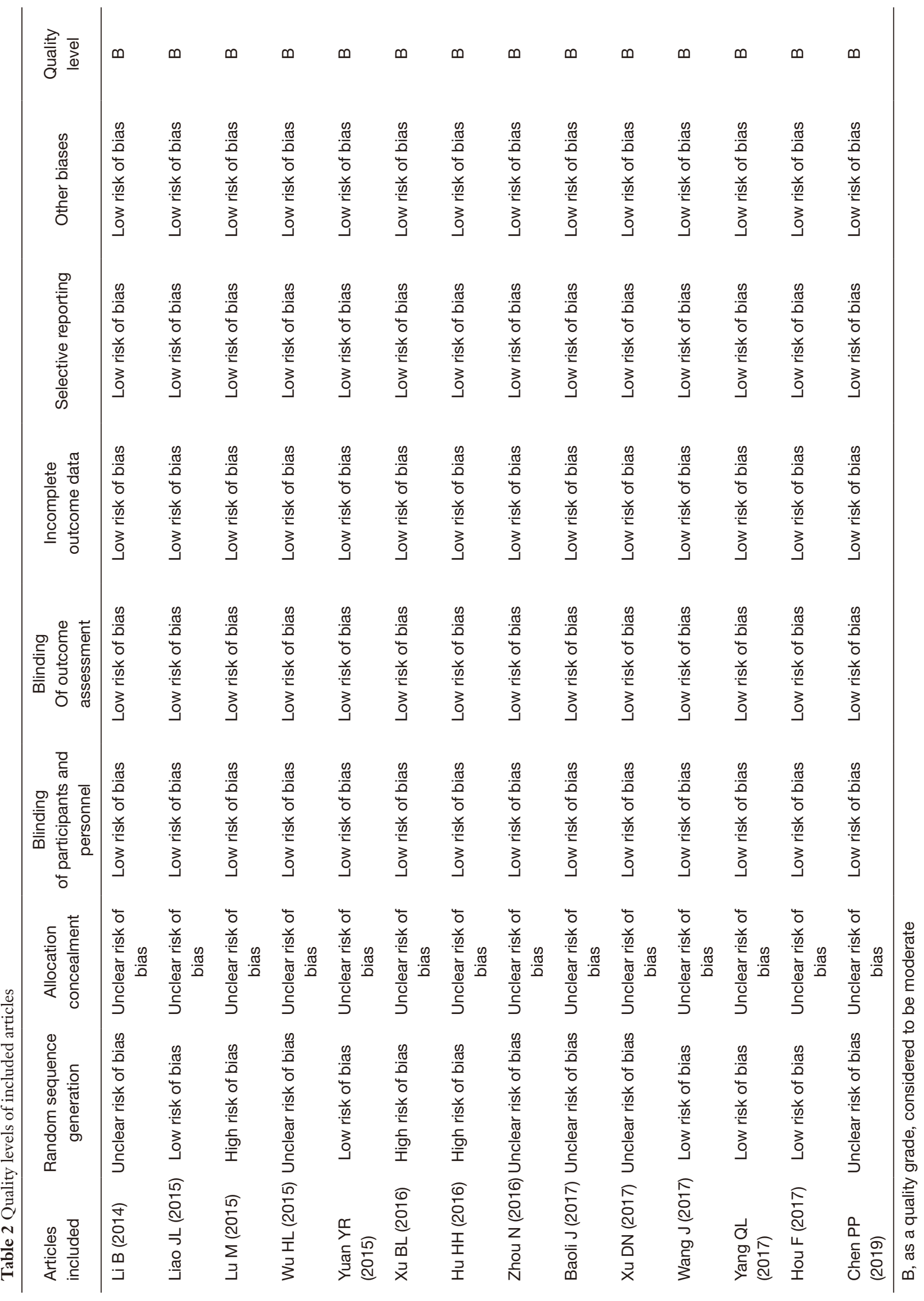




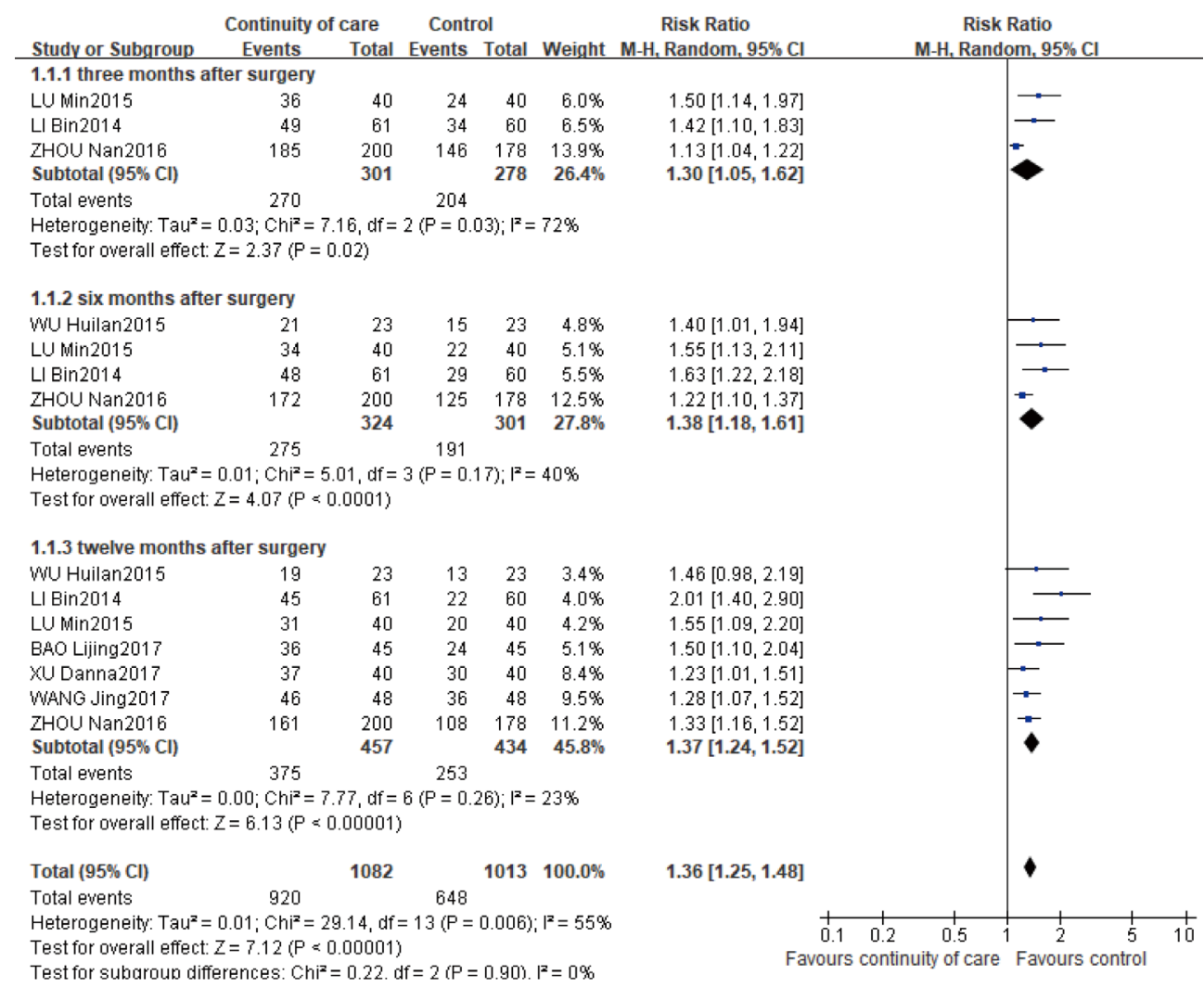

Figure 2 Forest plot for the impact of the continuity of care on anticoagulation therapy adherence.

anticoagulation adherence at 1 year postoperatively $(17,18,22)$. The results of the $\chi^{2}$ test showed considerable heterogeneity among the included studies $\left(\mathrm{I}^{2}>50 \%\right)$ and no statistical homogeneity $(\mathrm{P}>0.05)$; therefore, a randomeffects model was applied, which showed that the CC group had significantly better anticoagulation adherence than did the control group $[\mathrm{RR}=1.36,95 \% \mathrm{CI}:(1.25,1.48)$, $\mathrm{P}<0.00001$; Figure 2].

\section{Anticoagulant awareness}

In all, 3 of the included studies used anticoagulant awareness as an outcome measure $(10,11,16)$; of these, 2 reported patients' anticoagulant awareness at 3 months, 6 months, and 1 year postoperatively $(11,16)$, and one reported patients' anticoagulant awareness at 6 months postoperatively (10). The results of the $\chi^{2}$ test showed that there was no statistical heterogeneity among the included studies $(\mathrm{P}=0.74$, $\mathrm{I}^{2}=0 \%$, and therefore a fixed-effects model was applied, which showed that the CC group had significantly higher anticoagulant awareness than the control group $[R R=1.14$, 95\% CI: (1.09, 1.18), $\mathrm{P}<0.00001$; Figure 3].
Incidence of anticoagulation-associated complications and adverse event

In all, 9 of the included literature used the incidence of complications and adverse events associated with anticoagulation as an outcome measure; of these, 1 reported anticoagulation-associated complications and adverse events at 6 months and 1 year after discharge (12), 2 reported anticoagulation-associated complications and adverse events at 6 months after discharge $(13,15)$, and 6 reported anticoagulation-associated complications and adverse events at 1 year after discharge $(14,16,17,20-22)$. The results of the $\chi^{2}$ test showed that there was no statistical heterogeneity among the included studies $\left(\mathrm{P}=0.79, \mathrm{I}^{2}=0 \%\right)$, and therefore a fixed-effects model was applied, which showed that the CC group had significantly lower incidence of anticoagulationassociated complications and adverse events than did the control group $[\mathrm{RR}=0.24,95 \% \mathrm{CI}$ : $(0.17,0.35), \mathrm{P}<0.00001$; Figure 4].

\section{Quality of life}

In all, 4 articles $(14,17-19)$ used the quality of life (in 


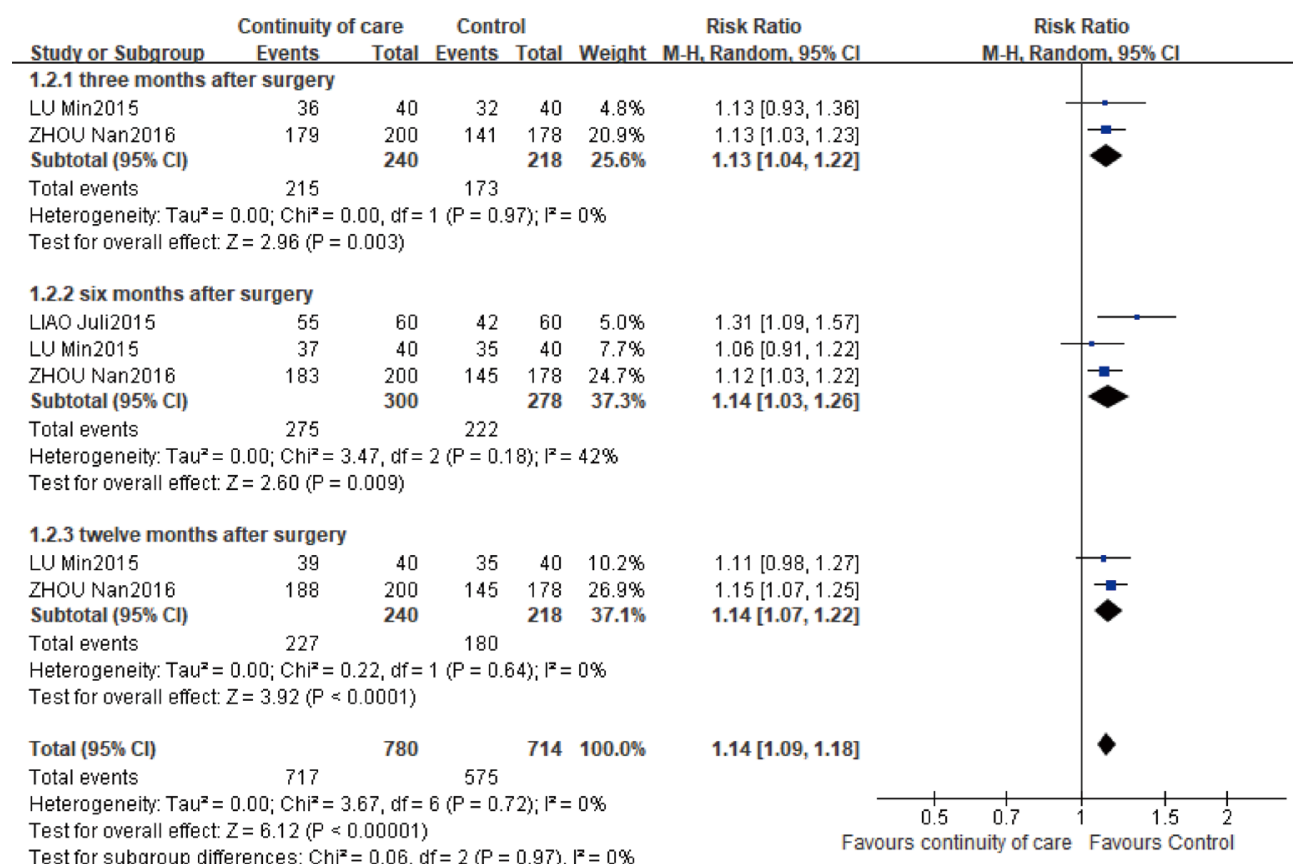

Figure 3 Forest plot for the impact of the continuity of care on anticoagulant awareness.

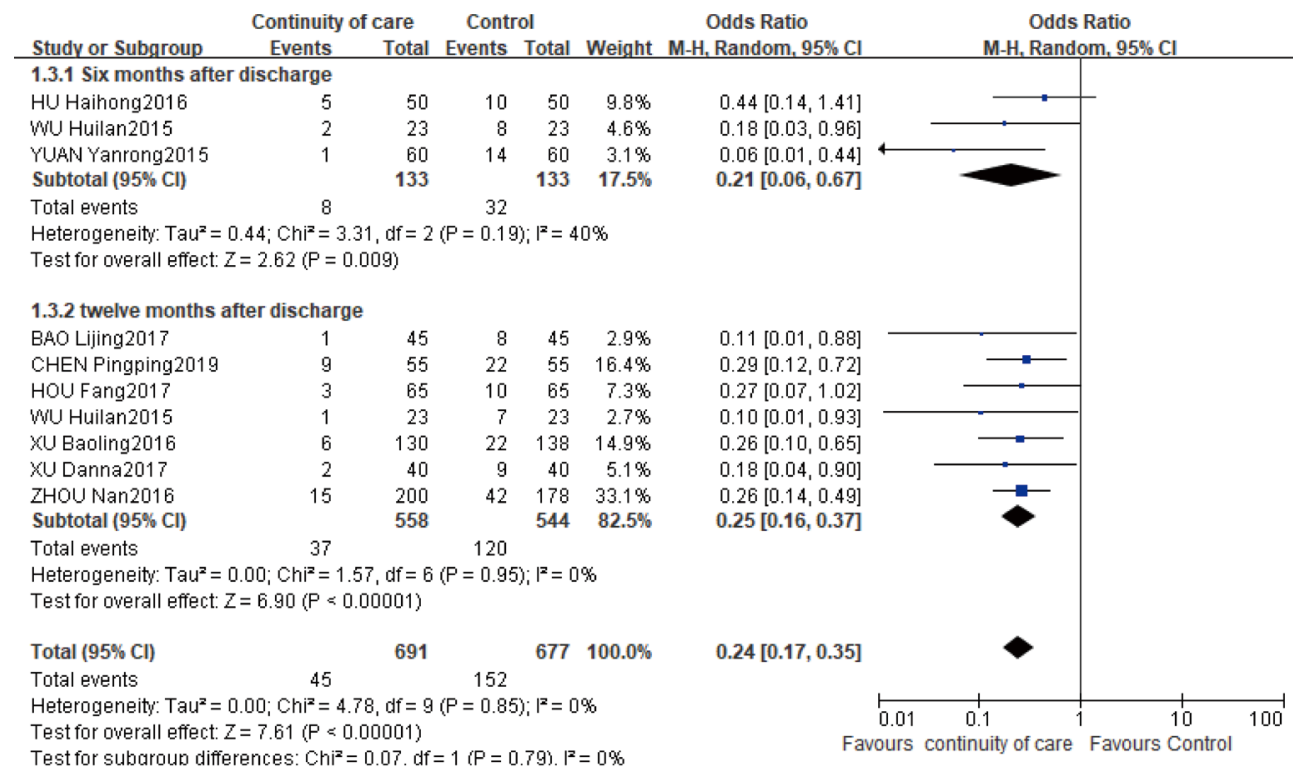

Figure 4 Forest plot for the impact of the continuity of care on the incidence of anticoagulation-associated complications and adverse events.

terms of physical domain, psychological domain, levels of independence, social relationships, and environment) as an outcome measure. The results of the $\chi^{2}$ test showed considerable heterogeneity among the included studies
$\left(\mathrm{I}^{2}>50 \%\right)$ and no statistical homogeneity $(\mathrm{P}>0.05)$; therefore, a random-effects model was applied, which showed that these 5 domains reflecting quality of life had significant differences between the two groups: physical domain [MD 


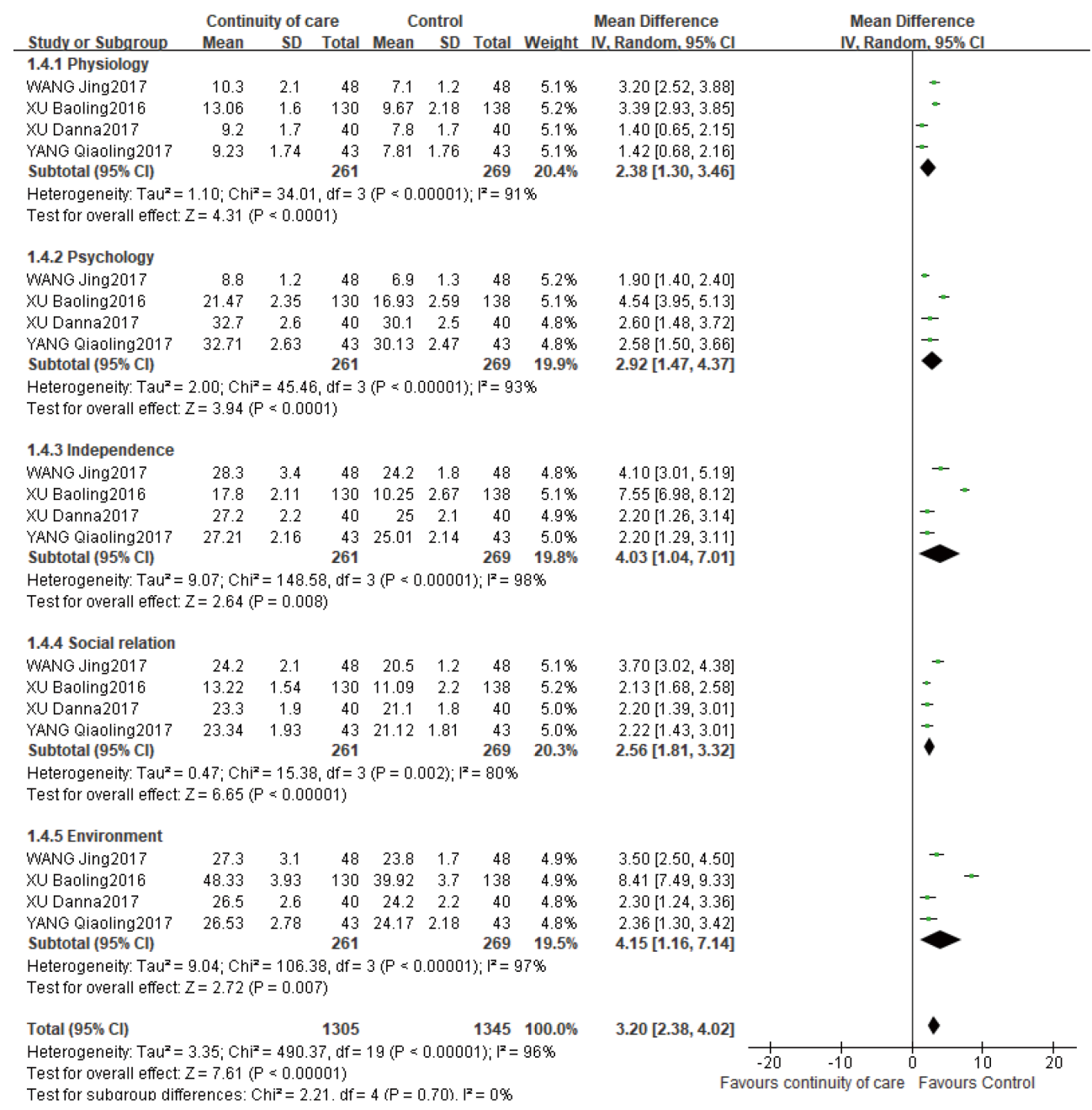

Figure 5 Forest plot for the impact of the continuity of care on quality of life.

$=2.38,95 \% \mathrm{CI}:(1.30,3.46), \mathrm{P}<0.0001]$, psychological domain $[M D=2.92,95 \% \mathrm{CI}:(1.47,4.37), \mathrm{P}<0.0001]$, levels of independence $[\mathrm{MD}=4.03,95 \% \mathrm{CI}:(1.04,7.01)$, $\mathrm{P}=0.008]$, social relationships $[\mathrm{MD}=2.56,95 \% \mathrm{CI}:(1.81$, $3.32), \mathrm{P}<0.00001]$, and environment $[\mathrm{MD}=4.15,95 \% \mathrm{CI}$ : (1.16, 7.14), $\mathrm{P}=0.007]$. Obviously, patients in the $\mathrm{CC}$ group had significantly higher quality of life than did those in the control group (Figure 5).

\section{Discussion}

\section{Methodological quality of the included literature}

This meta-analysis was performed to investigate the effect of CC on anticoagulation therapy and quality of life after
HVR. A total of 14 articles were included, all of which had a quality level of B. Overall, 5 articles described the randomization method and process, 5 did not elaborate on the grouping method, and 3 grouped the subjects in a nonrandomized manner (such as by time of admission or by the odd or even numbers of the admission numbers). Furthermore, 14 articles did not mention allocation concealment, and the risk of bias was evaluated as low because it was difficult to apply the blind method in discharged patients and the outcome indicators were not affected by the blind method. All 14 articles offered complete follow-up data, and there was no dropout of participants or withdrawal during the follow-up. The baseline data including gender and age were matched between the CC group and the control group. 


\section{Effectiveness of CC}

(I) Impact of CC on anticoagulation therapy adherence, anticoagulant awareness, and incidence of anticoagulation-associated complications and adverse events: As seen in the 14 included RCTs, CC helped to improve anticoagulation therapy adherence and anticoagulant awareness while lowering the incidence of anticoagulation-associated complications and adverse events. Bleeding and embolism due to inappropriate anticoagulation therapy is the most common life-threatening complication after HVR $(23,24)$. Due to the lack of knowledge and awareness concerning the disease, patients often cannot properly manage their disease or adjust their lifestyles, and their compliance with treatment protocols decreases with time after discharge, leading to the occurrence of anticoagulation-associated complications (9). Our meta-analysis showed that CC improved patients' compliance with anticoagulation therapy and raised their awareness of anticoagulants at 3 months, 6 months, and 1 year after discharge, and the compliance and awareness did not decrease with time after discharge; in addition, CC effectively reduced the incidence of anticoagulation-associated complications and adverse events at 6 months and 1 year after discharge.

(II) Impact of CC on quality of life: compared with the routine nursing practice, CC effectively improved the postoperative quality of life. However, the forest plot showed heterogeneity in all 5 domains (physical domain, psychological domain, levels of independence, social relationships, and environment) reflecting quality of life, which may be explained by the different severities of disease among participants in different studies or by the inconsistent CC interventions, intervention duration, and/or monitoring time points. Although the specific interventions of CC varied, they might include establishing a follow-up team of healthcare professionals, establishing and maintaining personal health record, offering health education on diseaserelated knowledge during hospitalization, developing discharge plans, and conducting post-discharge follow-up involving home visits and phone calls. It has been proposed that the quality of life after surgery is a dynamic process that will change with time and environment (25). As patients gradually recover from a surgery, their ability to take care of themselves increases, their confidence starts to be restored, and the quality of life gradually improves, which can reach a satisfactory level by 6 months after surgery (7). However, a survey outside of China revealed that patients were prone to fatigue and had low work and life abilities at 6 months after surgery, and most patients' mobility only recovered to about $55 \%$ of the normal range in the same age groups. The impact of CC on quality of life at different time points after interventions should be further investigated.

\section{Limitations}

The specific interventions and the content of health education were not completely consistent among different studies, which had an influence on the outcomes. There were no objective outcome indicators (such as blood pressure, blood lipids, or blood glucose) in this analysis. Despite the rapid development of CC in China in recent years, there were still many differences in nursing intervention measures compared with the relatively mature nursing intervention modes in foreign countries. Therefore, only Chinese-language articles were included in this analysis, and we did not perform a search for gray literature, which might have led to clinical heterogeneity.

\section{Conclusions}

CC can effectively improve patients' compliance with anticoagulation therapy and raise their awareness about medications, reduce the incidences of complications and adverse events, and thus improve the patients' quality of life. However, only a limited number of high-quality RCTs were included in our current analysis, and there are certain heterogeneities in the specific interventions, content of health education, and duration of intervention. Studies with more rigorous designs are warranted to further validate the impacts of CC on anticoagulation therapy adherence and quality of life after HVR.

\section{Acknowledgments}

Funding: This study was supported financially by Multidisciplinary centre of heart valve diseases in Zhongshan Hospital (No.:w2019-030) and Shanghai "New Star Medical Park" Young Medical Talents Training Funding Program (No.:Huwei Personnel [2020] No. 087). 


\section{Footnote}

Reporting Checklist: The authors have completed the PRISMA reporting checklist. Available at http://dx.doi. org/10.21037/apm-21-1167

Conflicts of Interest: All authors have completed the ICMJE uniform disclosure form (available at http://dx.doi. org/10.21037/apm-21-1167). The authors have no conflicts of interest to declare.

Ethical Statement: The authors are accountable for all aspects of the work in ensuring that questions related to the accuracy or integrity of any part of the work are appropriately investigated and resolved.

Open Access Statement: This is an Open Access article distributed in accordance with the Creative Commons Attribution-NonCommercial-NoDerivs 4.0 International License (CC BY-NC-ND 4.0), which permits the noncommercial replication and distribution of the article with the strict proviso that no changes or edits are made and the original work is properly cited (including links to both the formal publication through the relevant DOI and the license). See: https://creativecommons.org/licenses/by-nc-nd/4.0/.

\section{References}

1. Bland EF. Rheumatic fever: the way it was. Circulation 1987;76:1190-5.

2. Luo J, Lin S, Cai J, et al. Influence of Continuous Nursing Intervention on the Quality of Life of Patients after Cardiac Valve Replacement. Nursing Journal of Chinese People's Liberation Army 2017;34:70-3.

3. Wang B, Shen C, Huang L. Health education needs and guidance for patients with heart valve replacement. The Chinese Journal of Nursing 2003;38:229-30.

4. Zhang Y, Pi H. Research progress in the application of postoperative continuity care services. Chinese Journal of Modern Nursing 2010;16:3595-6.

5. Gong Y, Jin C, Zhang X, et al. Practice and effects of management for the transitional care team. The Chinese Journal of Nursing 2013;48:50-1.

6. Yin L, Yang J, Li C, et al. Effect of out-of-hospital continuity of care on prognosis of children with bronchial asthma in remission period. Nursing Practice and Research 2012;9:56-7.

7. Wen $Y$, Jiang X, Bai $Y$, et al. Research status of quality of life after heart valve replacement. West China Medical Journal 2016;31:1137-41.

8. Li Y, Yang X, Li H. SUPPORT Tools for evidenceinformed health Policymaking (STP) Appendix 2: Evaluation Designs (Adapted from the Cochrane Handbook for Systematic Reviews of Interventions). Chinese Journal of Evidence-Based Medicine 2010;10:647.

9. Li B, Duo L, Yu Y, et al. The effect of transitional care on the anticoagulant therapy compliance among discharged patients undergoing cardiac valve replacement surgery. Chinese Nursing Management 2014:1278-81.

10. Liao J. To study the effect of continuous nursing on the compliance of anticoagulant therapy in patients discharged from hospital after heart valve replacement. Medical Information 2015;28:143.

11. Lu M, Fei S. Effects of continuous nursing intervention on anticoagulant therapy compliance and quality of life of patients after heart valve replacement. Chinese Journal of Primary Medicine and Pharmacy 2015;24:3828-31.

12. Wu H, Zhou S, Xu L, et al. Influence of continuity of care on quality of life of patients after cardiac valve replacement. Chinese Nursing Research 2015:1303-6.

13. Yuan Y, Dong H, Hou F, et al. Effect of telephone followup on anticoagulant therapy compliance of patients after heart valve replacement. Journal of Qilu Nursing 2015;22:79-81.

14. Xu B, Su J, Li C, et al. The effect of extended care in rehabilitation of patients with cardiac valve replacement. J Nurs Admin 2016;16:278-80

15. Hu H, Gao W, Mao T, et al. Effect evaluation on continuous nursing care applied in anticoagulant management after mechanical valve replacement. Nursing and Rehabilitation Journal 2016;15:113-6.

16. Zhou N, Wang L, Ye F, et al. Study on Continuing Nursing to Improve the Compliance of Anticoagulant Therapy for Discharge Patients after Heart Valve Replacement. Journal of Taishan Medical College 2016;37:1073-4.

17. Xu D, Dai X. Effect of continuous nursing on quality of life of patients after heart valve replacement. Today Nurse 2017:38-40.

18. Wang J. Study of continuous nursing to improve the quality of life of patients after heart valve replacement. Journal of Clinic Nursing's Practicality 2017;2:32-4.

19. Yang $Q$. Effects of continuous nursing intervention on quality of life of patients after heart valve replacement. Journal of Clinical Medicine in Practice 2017;21:160-1, 164.

20. Hou F, Zhang Q, Cao X, et al. Effect of HtoH Continuous 
Care on Patients Undergoing Cardiac Valve Replacement. Journal of Qilu Nursing 2017;23:8-10.

21. Chen P, Ding X, Zhou N, et al. The application effect of continuous care after heart valve replacement. Journal of Huaihai Medicine 2019;37:103-5.

22. Bao L. Study on Continuing Nursing to Improve the Compliance of Anticoagulant Therapy for Discharge Patients after Heart Valve Replacement. Journal of Clinic Nursing's Practicality 2017;2:62-5.

23. Luo Y,Lin B, Chen X, et al. Effect of face to face health education on compliance with anticoagulant drugs in discharged patients after mechanical heart valve

Cite this article as: Yuan X, Chen Y, Zhuang Y, Qin W, Lin Y. Effect of continuity of care on anticoagulant therapy and quality of life after heart valve replacement: a systematic review and meta-analysis. Ann Palliat Med 2021;10(5):5568-5579. doi: 10.21037/apm-21-1167 replacement. Journal of Nursing Science 2010;25:81-3.

24. Wang Y, Wang Y, Liu F, et al. Influence of telephone follow-up on treatment compliance of patients underwent valve replacement: a meta-analysis. Chinese Journal of Geriatric Care 2014:12-5.

25. D'Antonio LL, Zimmerman GJ, Cella DF, et al. Quality of life and functional status measures in patients with head and neck cancer. Arch Otolaryngol Head Neck Surg 1996;122:482-7.

(English Language Editor: J. Gray) 